\title{
On Involutions
}

\section{O. Shisha* and C. B. Mehr**}

(December 7, 1966)

\begin{abstract}
Two methods are described of constructing real functions over the reals which are one-to-one, assume every real value and are their own inverses, and several examples are given. It is also shown that such a function, if everywhere continuous, is either the function $f(x) \equiv x$ or else is strictly decreasing.
\end{abstract}

Key Words: Inverses, involutions, real functions.

1. We shall consider real functions $f$ whose domain is the set of real numbers, which take on every real value, are one-to-one, and satisfy for every real $x$, $f^{-1}(x)=f(x)$, where $f^{-1}$ is the inverse function of $f$. We denote by $I$ the set of all such functions. Recall that functions which are their own inverses are called involutions.

Suppose that a real function $f$ has as its domain the set of real numbers. Then it belongs to $I$ if and only if

$$
f(f(x)=x \text { for every real } x .
$$

Indeed, if $f \epsilon I$, then for every real $x, f(f(x))=f\left(f^{-1}(x)\right)$ $=x$. Conversely, if (1) holds, then $f$ takes on every real value, is one-to-one (for $f\left(x_{1}\right)=f\left(x_{2}\right)$ implies $\left.x_{1}=f\left(f\left(x_{1}\right)\right)=f\left(f\left(x_{2}\right)\right)=x_{2}\right)$, and for every real $x, f^{-1}(x)=f(x)$.

Note that the graph of every $f$ in $I$ is symmetric in the line $y=x$.

Conversely, if $G$ is a set in the $x, y$ plane, symmetric in the line $y=x$ and containing, for every real $x$, a unique point whose abscissa is $x$, then $G$ is the graph of a function belonging to $I$.

2. One way of obtaining functions in $I$ is the following. Start with a real function $g(x, y)$ whose domain is the set of all ordered pairs of real numbers, and which is such that $g(x, y)=0$ implies $g(y, x)=0$. (This property holds, e.g., if $g$ is symmetric, i.e., if for every real $x, y$, we have $g(y, x)=g(x, y)$.) Suppose that for every real $x$, there is a unique real $y$ (to be denoted $f(x))$ such that $g(x, y)=0$. Then $f$ (with domain the set of reals) belongs to $I$. Indeed, for every real $x$,

$$
g(f(x), x)=g(x, f(x))=0,
$$

and consequently $f(f(x))=x$.

\footnotetext{
*Aerospace Research Laboratories, Wright-Patterson AFB, Ohio 45433.

**The contribution of this author was made while he was working at Aerospace Research Laboratories, Wright-Patterson AFB, Ohio. Present address: Ohio University, Athens, Ohio 45701 .
}

EXAMPLE 1. Let $g(x, y) \equiv x+y-c, c$ being an arbitrary real constant. We obtain from it the function $f(x) \equiv c-x$ belonging to $I$.

ExAMPLE 2. Let $g(x, y) \equiv x-y$. The corresponding $f \in I$ is $f(x) \equiv x$.

EXAMPLE 3 . Let $g(x, y) \equiv x^{3}+y^{3}-c, c$ being an arbitrary real constant. We get from it the function $f(x) \equiv \sqrt[3]{c-x^{3}} \epsilon I$.

3. Another method of obtaining functions in $I$ is based on the last paragraph of section 1 . We illustrate this method by the following

EXAMPLE 4 . In the $X, Y$ plane consider the hyperbola $X^{2}-\frac{1}{2} Y^{2}=1$. Let $R, L$ denote, respectively, its righthand and left-hand branches. Consider now a new coordinate system, $x, y$, obtained from the $X, Y$ system by a clockwise rotation of $45^{\circ}$. In the new coordinate system the equation of $R$ (which is symmetric in the line $y=x$ ) is

$$
y=-3 x+2\left(2 x^{2}+1\right)^{1 / 2} .
$$

Thus, $f(x) \equiv-3 x+2\left(2 x^{2}+1\right)^{1 / 2}$ belongs to $I$. Similarly, the equation of $L$ in the new coordinate system is

$$
y=-3 x-2\left(2 x^{2}+1\right)^{1 / 2},
$$

and consequently, $f(x) \equiv-3 x-2\left(2 x^{2}+1\right)^{1 / 2}$ belongs to $I$.

4. Consider the functions in $I$ which are everywhere continuous. Since such a function takes on every real value exactly once, it must be, throughout the real line, either strictly increasing or strictly decreasing. For example, $f(x) \equiv x$ is a function in $I$ which is strictly increasing. It is interesting to note that all other everywhere continuous functions in I are strictly decreasing. Indeed, let $F(x)(\neq \equiv x)$ be an everywhere continuous function in $I$. Then its graph contains two points which do not lie on the line $y=x$, but which are symmetric in this line. Let $\left(x_{1}, y_{1}\right),\left(x_{2}, y_{2}\right)$ (with $x_{1}<x_{2}$ ) be such points. Then $y_{1}>y_{2}$ (draw a figure!). So $F\left(x_{1}\right)>F\left(x_{2}\right)$, and consequently, $F$ is strictly decreasing. 
5. Let us examine the smoothness of the various examples we have of functions belonging to $I$. The functions $c-x$ and $x$ of Examples 1 and 2 are differentiable throughout the real line; in fact they are analytic at each real point. The function $f(x) \equiv \sqrt[3]{c-x^{3}}$ of Example 3 is everywhere continuous. If $c=0$, it reduces to $-x$. Otherwise, it is everywhere differentiable except at the point $x=\sqrt[3]{c}$, where it is not.
Let us now look at the functions $f$ of Example 4. The function $2 z^{2}+1$ of the complex variable $z$ vanishes at $2^{-1 / 2} i,-2^{-1 / 2} i$ and nowhere else. Consequently, the real functions $-3 x+2\left(2 x^{2}+1\right)^{1 / 2},-3 x-2\left(2 x^{2}+1\right)^{1 / 2}$ of Example 4, are analytic at every point of the $x$ axis.

(Paper 71B1-192) 